\title{
Editorial
}

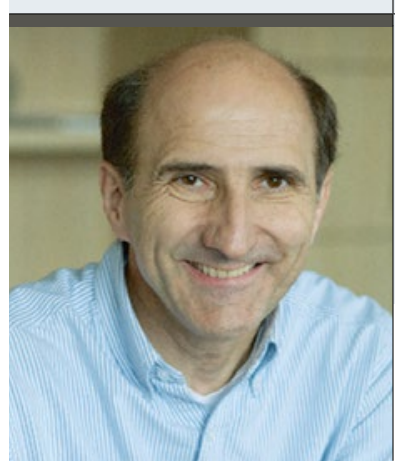

"Der Wissenschaft wäre sehr gedient, wenn sie sich mehr der

grundlegenden wissenschaftlichen Vorgehensweise erinnern

würde."

Prof. Dr. med. Ralf Ihl

Maria-Hilf-Krankenhaus,

Klinik für Gerontopsychiatrie und -psychotherapie, Krefeld

\section{Jahre Alzheimer und ein bisschen weiser?}

A lzheimer publizierte 1906 seine am Gehirn von Auguste Deter gewonnenen Erkenntnisse als ,eigenartige Erkrankung der Hirnrinde" und lenkte so das Augenmerk auf eine frühe Form der schon Jahrtausende zuvor von Seneca beschriebenen „senilen Demenz“. Sein Lehrer Kraepelin nahm das zum Anlass, die frühe Form der senilen Demenz „Alzheimer-Krankheit“ zu nennen. Bis in die zweite Hälfte des letzten Jahrhunderts geschah dann recht wenig. Versuche, die Krankheit von „Altersblödsinn“ und „Presbyophrenie“ abzugrenzen, blieben erfolglos. Lange teilte schließlich 1935 mit, die Alzheimer-Krankheit sei selten. In den 1960er-Jahren wurde der Unterschied zwischen früher und später Form durch die Wissenschaft aufgegeben. Was die Häufigkeit angeht, könnte Lange recht behalten. Wie vor einem Jahr an gleicher Stelle beschrieben, fanden sich die klassischen Marker, die heute für die Alzheimer-Krankheit sprechen, bei Auguste Deter nicht.

\section{Irrungen und Wirrungen der frühen Forschungsarbeit ...}

Trotz unscharfer Definition der Krankheit wurde viel geschrieben und geforscht. Der Hinweis auf die aus unscharfer Definition resultierende Heterogenität der Gruppen und die daraus resultierende eingeschränkte Beurteilbarkeit von Ergebnissen wurde allenfalls als störend wahrgenommen. Es ist zumindest nicht auszuschließen, dass so das Resultat unbefriedigend bleibt: Die Krankheit kann auch heute noch weder geheilt noch gestoppt werden. Die durchgemachten Irrungen und Wirrungen der Wissenschaft bei der Erforschung der Krankheit könnten uns aber einiges für die Zukunft lehren.

Ein paar Irrwege seien kurz beleuchtet, als erstes die Geschichte der falschen Spur. In Zellpräparaten von Menschen, die zuvor Demenzsymptome gezeigt hatten, wurde das giftige Aluminium gefunden. So- fort war klar, dass das früher verwendete Aluminiumgeschirr für die Krankheit verantwortlich sein muss. Kritische Anmerkungen, dass nicht jeder Mensch, der dialysepflichtig ist und einen hohen Aluminiumspiegel hat, auch eine Demenz entwickelt, wurden in den Wind geschlagen. Es folgten Studien zum Aluminium im Trinkwasser bei Millionen von Menschen und aufgrund der immensen "Teilnehmerzahlen“ wurde selbst ein minimaler Unterschied an Erkrankungen zwischen den Gruppen signifikant. Die Idee fand ein jähes Ende, als sich herausstellte, dass das Aluminium mit der Färbung der Nervenzellen in sie hineingebracht worden war. Das Ergebnis: Aluminium ist nicht gut für den Menschen, aber doch nicht die Ursache der Demenz.

Auch Statistik widerlegt falsche Annahmen nicht von selbst. Kaum besser das unaufmerksame Rechnen, dass Rauchen vor Demenz schützen sollte, da viel weniger Raucher unter den Demenzkranken zu finden waren, bis auffiel, dass diese vielleicht schon zuvor an den durch das Rauchen bedingten Krankheiten verstorben sein könnten.

Unverwüstlich schien über lange Zeit die Amyloidhypothese. Nachdem sich das Amyloid aus Gehirnzellen entfernen ließ und die Krankheit trotzdem blieb, hat sie etwas Schaden gelitten. Aufgeben kennt die Wissenschaft nicht. Man könnte schließen, nicht alles, was das Denken ändern müsste, ändert das Denken.

\section{... die Lehren für die Forschungsarbeit heute}

Mit der Frage, warum bei der Unmenge von Studien, zirka 30.000 pro Jahr, nichts Brauchbares herauskommt, tut sich die Wissenschaft sehr schwer. Es hakt oft schon beim Ansatz von Studien. Die schon angeklungene Heterogenität der Gruppen ist bekannt. Sie wandelt sich aber nicht von allein in Homogenität. Untaugliche Instrumente für das $\mathrm{Zu}-$ sammenstellen von Gruppen werden eifrig weiter 


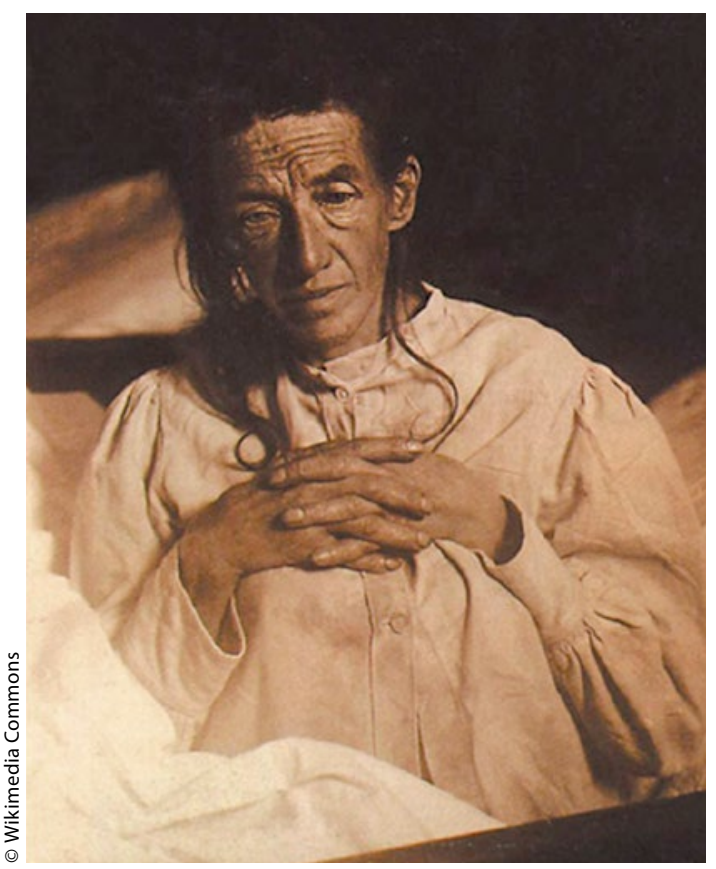

Die klassischen Marker, die heute für die AlzheimerKrankheit sprechen, fanden sich bei Auguste Deter nicht.

genutzt. Durch gute Öffentlichkeitsarbeit kennt zwar mittlerweile nahezu jeder den Mini-MentalStatus. Kaum einer hat jedoch die Vielzahl von Studien wahrgenommen, die belegen, dass er zu nichts taugt, und so wird er weiter munter zur Diagnostik, Gruppenbildung und sogar zur Therapieerfolgsmessung eingesetzt, obwohl schon ein Jahr nach seiner Veröffentlichung bessere Tests publiziert wurden.

\section{Administrierte Datenbank anstelle Kaffeesatzlesen}

Nachlesen ist sicherlich nicht die aktuelle Stärke der Wissenschaft. Wie auch? Bei 30.000 Artikeln im Jahr ist das schier unmöglich. Aber was wäre Wissenschaft ohne eine Lösung. Ein erster Ansatz waren Reviews. Mit etwas Glück hat der Autor persönlich die Artikel zumindest gelesen, der Reviewer mit an Sicherheit grenzender Wahrscheinlichkeit nicht. Oft sind dabei schon die Stärken und Schwächen von Studien verloren gegangen oder nicht wahrgenommen worden. Ein Lösungsansatz, wie genauer zu prüfen wäre, kommt bei Zeitmangel nicht infrage. Die Flucht nach vorne sind Reviews von Reviews mit genialen Begründungen, warum das viel besser ist, beispielsweise: „Falsches verliert sich in der Menge der Daten“. Das Ergebnis beinhaltet einen Beliebigkeitsgrad, der an Kaffeesatzlesen erinnert. Der Metaanalyseansatz scheitert häufig an denselben Schwächen. Wir wissen, dass Reviewer selbst nach Training nur einen Bruchteil der Fehler einer
Studie erkennen. So werden wir auch in Zukunft mit unseren Schwächen leben müssen.

Die fehlende Kenntnis und Sauberkeit in der Methodik von Wissenschaftlern ist sicherlich ein wesentlicher Grund des schleppenden Vorankommens. Die Konsequenzen für die Therapie erleben wir tagtäglich. Für die Kranken bedeutet das nach der aktuellen Leitlinie, dass die Diagnostik sehr differenziert die Störungen beschreibt, die Ursache der Krankheit im Dunkeln bleibt und gerade einmal fünf Medikamente bei einzelnen Menschen, für eine begrenzte Zeit einen moderaten Effekt auf die Kognition zeigen. Für Verhaltensauffälligkeiten reichen die Studiendaten nicht aus, um ein Medikament zu empfehlen.

Die fehlenden Ergebnisse im biologischen Bereich können sicher durch den psychosozialen Bereich nicht ausgeglichen werden. Da psychosoziale Erkenntnisse für die Kranken und ihre Angehörigen eine unmittelbare Erleichterung bringen, ist es aber umso erfreulicher, dass in letzter Zeit auch hier die Erkenntnis Einzug hält, dass Dinge zweimal klappen sollten, um nützlich für andere zu sein.

Der Wissenschaft wäre sehr gedient, wenn sie sich mehr der grundlegenden wissenschaftlichen Vorgehensweise erinnern würde. Wenn es schon unmöglich ist, als einzelner Forscher die Literaturflut zu überblicken, benötigt es intelligente Wege des Exzerpierens. Das wissenschaftliche Chaos könnte beispielsweise beendet werden, wenn eine öffentlich verfügbare und administrierte Datenbank, Erkenntnisse nachlesbar und mit Kommentarfunktion erfassen und zusammenfassen würde. Der Grundstein dafür wurde im Kompetenznetz Demenz bereits vor vielen Jahren gelegt. Die Idee wurde aus Kostengründen nicht weiterverfolgt. Zu kurz gedacht. Ein neuer Aufschlag wäre hilfreich.

Unabhängig davon arbeitet die Wissenschaft weiter. Einige interessante Ergebnisse finden Sie in der vor Ihnen liegenden Ausgabe von DNP - Der Neurologe \& Psychiater.

Viel Freude beim Lesen wünscht Ihnen

\section{Ralf Ihl}

